\title{
Drenaje de las Venas Facial, Lingual y Tiroidea Superior en el Hombre
}

\author{
Drainage of Facial, Lingual and Superior Thyroid Veins in Humans \\ "Luiz Carlos Buarque Gusmão; **Célio Fernando de Sousa-Rodrigues; \\ ${ }^{* * * *}$ Fabiana Sophia Gonzalez da Nóbrega \& ${ }^{* * * *}$ Lígia Maria Teixeira Pereira da Silva
}

GUSMÃO, L. C. B.; SOUSA-RODRIGUES, C. F.; NÓBREGA, F. S. G. \& PEREIRA DA SILVA, L. M. T. Drenaje de las venas facial, lingual y tiroidea superior en el hombre. Int. J. Morphol., 24(4):685-688, 2006.

RESUMEN: La gran diversidad de descripciones acerca de las afluencias de las venas facial, lingual y tiroidea superior en el hombre, estas presentan una serie de controversias entre los autores investigados. Buscando ofrecer un patrón para la formación de troncos venosos a partir de las referidas venas, los autores realizaron la disecación de 42 faces laterales del cuello de cadáveres humanos adultos, de ambos sexos, con edades variadas y fijados en formaldehido a 10\%. Se observó la formación de troncos venosos en 59,5\% de los casos, habiendo ocurrido formación del tronco tiroilingofacial en 38,1\%, del tronco lingofacial en 14,2\%, del tronco tiroilingual en 4,8\% y del tronco tiroilingofaringofacial en apenas $2,4 \%$. No fue encontrada la formación del tronco tiroifacial descrito en la literatura. En los $40,5 \%$ de los casos en los que no hubo formación de troncos venosos, las venas presentaron desembocaduras solitarias.

PALABRAS CLAVE: Vena facial; Vena lingual; Vena tirodea superior; Anatomía; Drenaje venoso.

\section{INTRODUCCIÓN}

La formación venosa de la región cervical deriva embriológicamente de una red vascular subcutánea, donde algunas de sus mallas se alargan de manera no uniforme, generando los vasos venosos; de ahí su gran variabilidad.

Las venas facial, lingual y tiroidea superior presentan una gran diversidad de descripciones, principalmente cuanto a sus afluencias. Las venas pueden confluir entre sí, promoviendo la formación de un tronco venoso único, denominado tronco tirolinguofacial (Testut,1924; Testut \& Jacob, 1958; Alves 1962; Pauchet \& Dupret, 1965; Testut \& Latarjet, 1968; Sneel, 1984; Brandão \& Ferraz, 1989; Latarjet \& Ruiz Liard, 1993). Este tronco es descrito en la literatura consultada con frecuencias de 10\% (Madeira \& Hetem y Gonçalves et al., 1990); 30\% (Bruno) y 44\% (Ghezzi, 1971).

Las venas tiroidea superior y lingual se pueden unir, formando el tronco venoso tirolingual (Bruno, 1924; Ghezzi; Madeira \& Hetem, 1971; Putz \& Pabst, 1995). El tronco linguofacial (Bruno; Ghezzi; Osírio, 1957; Madeira \& Hetem; Sicher et al., 1977; Ferner \& Staubesand, 1980; Goss, 1988; Moore, 1995; Putz \& Pabst; Shima et al., 1998; Wiliams et al., 1995) se origina de la unión entre las venas lingual y facial. Ya el tronco tirofacial resulta de la junción entre las venas tiroidea superior y facial (Bruno; Ghezzi; Prives et al., 1971; Madeira \& Hetem; Wiliams et al.; Shima et al.) y fue encontada en la literatura con frecuencia de 1,4\% (Madeira \& Hetem), 2\% (Ghezzi), 4\% (Bruno) y 6,9\% (Prives et al., 1971).

En la literatura se encontró la formación de troncos venosos en $100 \%$ de los casos ( Bruno \& Ghezzi); en 62,9\% (Madeira \& Hetem) y en 89,7\% (Shima et al.).

Por otro lado, puede no haber la formación de troncos venosos, teniendo las venas facial, lingual y tiroidea superior afluencias independientes (Lockhart et al., 1965; Goss; Gardner et al., 1988; Spalteholtz, 1988).

* Profesor Adjunto del Departamento de Morfología de la Universidad Federal de Alagoas; Master Doctor en Anatomía Humana; Miembro Titular del Colegio Brasileño de Cirujanos, Brasil.

** Profesor Adjunto del Departamento de Morfología de la Universidad Federal de Alagoas; Master Doctor en Anatomía Humana, Brasil.

*** Médica Residente de Cirugía General del Hospital Universitario de la Universidad Federal de Alagoas, Brasil.

***** Monitora de la Disciplina de Anatomía Humana de la Universidad Federal de Alagoas, Brasil.

Trabajo realizado en el Departamento de Morfología de la Univesidad Federal de Alagoas y financiado por el Programa Institucional de Becas de Iniciación Científica - PIBIC del CNPq 
A causa de la discordancia encontrada en la literatura investigada respecto de la formación de los troncos originados por la unión de las venas facial, lingual y tiroidea superior, el presente estudio tiene por fin describir patrones de formación de los troncos venosos a partir de las referidas venas, ofreciendo soporte anatómico para los procedimientos quirúrgicos en que se involucran estas estructuras vasculares.

\section{MATERIAL Y MÉTODO}

Para la realización del presente estudio fueron utilizadas 42 faces laterales del cuello de cadáveres humanos adultos, de ambos sexos, fijados en formaldehido a $10 \%$ y previamente disecados por los alumnos del curso médico de la Universidad de Alagoas y de la Escuela de Ciencias Médicas de Alagoas, Brasil.

Las regiones cervicales fueron sometidas a una disecación minuciosa, teniendo por finalidad identificar las venas facial, lingual y tiroidea superior y seguirlas hasta sus desembocaduras, así como, observar la formación de troncos venosos por estas venas.

\section{RESULTADOS}

En nuestras observaciones constatamos la formación de troncos venosos a partir de las venas facial, lingual y tiroidea superior en 59,5\% de los casos. Se encontró un tronco formado por la unión de las venas facial, lingual y tiroidea superior (tronco tirolinguofacial) en 38,1\% (16/42) de los casos (Fig. 1). En 6 casos (14,2\%) se verificó la formación del tronco linguofacial (Fig. 2). Hubo formación del tronco tirolingual (Fig. 3) en 2 casos (4,8\%) y en sólo 1 caso $(2,4 \%)$ se constató la existencia de un tronco venoso tirolinguofaringofacial (Fig. 4). No fue encontrada la formación del tronco tirofacial.

Las venas investigadas se presentan con afluencias independientes en 40,5\% de nuestra casuística.

\section{DISCUSIÓN}

En el presente estudio constatamos que pocos autores describieron de modo estadístico la formación de troncos venosos, entre ellos Bruno; Ghezzi; Madeira \& Hetem y Prives et al.

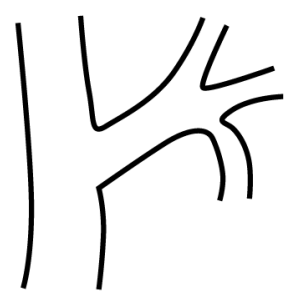

Fig. 1. Tronco tirolinguofacial. $38,1 \%$

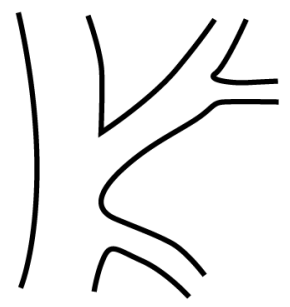

Fig. 2. Tronco linguofacial. 14,2\%

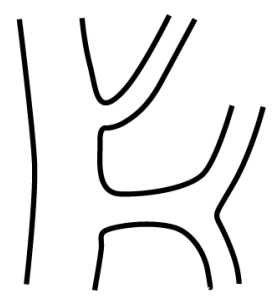

Fig. 3. Tronco tirolingual. $4,8 \%$

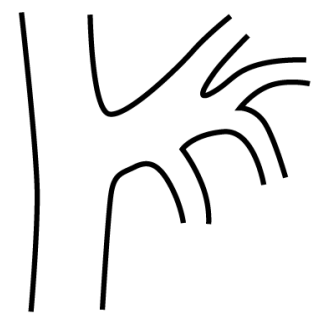

Fig. 4. Tronco tirolinguofaringofacial. 2,4\%

En nuestras disecaciones encontramos la formación de troncos venosos en 59,5\% de los casos, lo que nos lleva a cuestionar la afirmación hecha por Bruno y Ghezzi quienes observaron formación de troncos venosos en todos los casos por ellos disecados. Tal divergencia puede ser explicada debido a la gran variación en la formación de la red venosa cervical, como también por la utilización de pequeñas muestras por estos autores, necesitando, en este caso, de muestras mayores para el establecimiento de datos estadísticos sólidos y confiables. 
Detectamos la formación del tronco tirolinguofacial en $38 \%$ de los casos, a diferencia de la reducida frecuencia de $10 \%$ encontrada por Madeira \& Hetem y Gonçalves, 1990.

En nuestras piezas anatómicas no fue encontrada la presencia del tronco tirofacial, a pesar de que el mismo fue referido con frecuencias que varían de 1,4\% (Madeira \& Hetem) a 6,9\% (Prives et al., 1971).

Fundamentados en nuestros descubrimientos, con- cluimos que hay una gran variabilidad para los patrones de confluencia venosa entre las venas facial, lingual y tiroidea superior. De igual manera, la falta de uniformidad entre las descripciones encontradas en la literatura acerca de la formación venosa cervical se explica por el hecho de que las venas de esa región son derivadas embriológicamente de una red venosa subcutánea, donde algunas mallas se alargan de manera no uniforme, originando los vasos venosos; tal hecho, segundo Sicher, justifica esa gran diversidad.

GUSMÃo, L. C. B.; SOUSA-RODRIGUES, C. F.; NÓBREgA, F. S. G. \& PEREIRA DA SILVA, L. M. T. Drainage of facial, lingual and superior thyroid veins in humans. Int. J. Morphol., 24(4):685-688, 2006.

SUMMARY: Despite the great diversity of descriptions concerning the inflows of the facial, lingual and superior thyroid veins in man, they present important controversies between the studied authors. Aiming to supply a pattern for the formation of venous trunks by the cited veins, the authors analyzed 42 lateral faces of dissected adult human cadavers of both genders, different ages and preserved in a $10 \%$ solution of formaldehyde. The study revealed the formation of venous trunks in $59,5 \%$ of the cases, the thyroid-lingual-facial trunk appears in $38,1 \%$, the lingual-facial trunk in $14,2 \%$, the thyroid-lingual trunk in $4,8 \%$ and the thyroid-lingual-pharyngeal-facial trunk in just $2,4 \%$. It was not found the formation of thyroid-facial trunk described in the analyzed literature. $40,5 \%$ of the cases appears with no venous trunk formation, in those situations the veins end alone.

KEY WORDS: Facial vein; Ligual vein; Superior thyroid vein; Anatomy;

\section{REFERENCIAS BIBLIOGRÁFICAS}

Alves, E. Anatomia Topográfica. 2ª ed. Rio de Janeiro, 1962.

Brandão, L. G.; Ferraz, A. R. et al. Cirurgia de cabeça e pescoço. São Paulo, Roca, 1989. p. 565.

Bruno G. Recerche Col Método Statístico sella Morfologia della Facia e Del Collo nell' Uomo. Lê Vene dell' Adulto. Ach. Ital. Anat. Embriol, 21:273-93, 1924.

Ferner, H. \& Staubesand, J. Trattato di Anatomia Umana Funcionale. Padova, Ed. Piccin, 1980, V. 2. p 490.

Gardner, E.; Gray, D. \& O’ Rahilly, R. Anatomia. $4^{\mathrm{a}}$ ed. Rio de Janeiro, Guanabara Koogan, 1988.

Ghezzi, W. Contribuição ao estudo do sistema jugular (veias jugulares interna, externa e anterior). 1947; Tese de Doutorado Livre, Faculdade de Medicina da Universidade de Porto Alegre.

Gonçalves, D. R.; Landucci, C.; De Toledo Ramalho, L. R. \& Porciúncula, H. F. Study of the cervical portion of the facial vein of fetuses, newborns and childres. Rev. Odontol. UNESP., 19 (1):21-9, 1990.

Goss, C. M. Gray Anatomia. 29ª ed. Rio de Janeiro, Guanabara Koogan, 1977.
Latarjet, M. \& Ruiz Liard, A. Anatomia Humana. $2^{\text {a }}$ ed. São Paulo, Panamericana, 1993. pp 1137-1138.

Lockhart, R. D.; Hamilton, G. F. \& Fyfe, F. W. Anatomia do Corpo Humano. Cidade do México, Interamericana, 1965. p 638.

Madeira, M. C. \& Hetem, S. Dados Morfológicos sobre as veias retromandibular, facial, facial comum, jugular anterior e tronco tireo-lingo-facial em fetos e crianças. Arg. Anat. Antropol, 35:211-242, 1971.

Moore, K. L. Anatomia Orientada Para a Clínica. $3^{\mathrm{a}}$ ed. Rio de Janeiro, Guanabara Koogan, 1995, pp 607-75.

Osírio, P. Anatomia Aplicada da cabeça. Rio de Janeiro, Científica, 1957, pp 325-332.

Pauchet, V. \& Dupret, S. Atlas de Anatomia. $5^{\mathrm{a}}$ ed. Rio de Janeiro, Atheneu, 1965. p 140.

Prives, M.; Lisenkov, N. \& Bushkovich, V. Anatomia Humana. Ed. Mir, Moscu, 1971. V. 2. pp. 105-107.

Putz, R. \& Pabst, R. Sobota Atlas de Anatomia Humana. 20ª ed. Rio de Janeiro, Guanabara Koogan, 1995, pp 77754. 
Shima, H.; von Luedinghausen, M.; Ohno, R. \& Mighi, K. Anatomy of microvascular anastomosis in the neck. Plast. Reconstr. Surg. Jan, 101(1):33-41, 1998.

Sicher, H.; Dubrul, E. L. \& Picosse, M. Anatomia Bucal. $6^{\text {a }}$ ed. Rio de Janeiro, Guanabara Koogan, 1977. pp. $337-$ 9.

Snell, R. S. Anatomia. $2^{\text {a }}$ ed. Rio de Janeiro, Medsi, 1984,pp 564-5.

Spalteholtz, W. Atlas de Anatomia Humana. São Paulo, Roca, 1988. V. 2. pp 140-3.

Testut, L. Traité d'Anatomie Humaine. Angéologie- Système Nerveux Central. $2^{\mathrm{a}}$ ed. Paris, Librarie Octave Doin, 1924. V. 2. pp. $314-21$.

Testut, L. \& Jacob, O. Compendio de Anatomia Topográfica. $11^{a}$ ed. Barcelona, Salvat, 1968. pp. 471-2.

Testut, L. \& Latarjet, A. Tratado de anatomia humana. $9^{\mathrm{a}}$ ed. Barcelona, Salvat, 1968. pp. 471-472.

Williams, P. L.; Warwick, R.; Dyson, M. \& Bannister, L. H. Gray Anatomia. 37 a ed. Rio de Janeiro, Guanabara Koogan, 1995, V. 2. pp 745-8.
Dirección para correspondencia:

Prof. Dr. Luiz Carlos Buarque

Condominio Aldebaran Alfa

Quadra F, no 08. Tabuleiro dos Martins

CEP: $57080-900$.

Maceió-AL.

BRASIL

Telefonos: 55-82-9983-9161

$55-82-3358-5931$

Recibido : 15-08-2006

Aceptado: 19-10-2006 